\title{
Estudos ecogeográficos de Eplingiella fruticosa (Salzm. Ex Benth. Harley\& J.F.B. Pastore): Uma espécie medicinal do semiárido do Brasil
}

\author{
Ecogeographic studies on Eplingiella fruticosa (Salzm. Ex Benth. Harley \& J.F.B. Pastore: A \\ medicinal species of the semiarid region of Brazil \\ Estudios ecogeográficos del Eplingiella fruticosa (Salzm. Ex Benth. Harley \& J.F.B. Pastore): Una \\ especie medicinal de la región semiárida de Brasil
}

Recebido: 16/03/2021 | Revisado: 23/03/2021 | Aceito: 04/04/2021 | Publicado: 15/04/2021

Edvan Assis de Oliveira
ORCID:https://orcid.org/0000-0002-9114-6851
E-mail: eo4001 @ gmail.com
Lenaldo Muniz de Oliveira
ORCItuto Federal de Educação, Ciência Te Tho
ORttps://orcid.org/0000-0002-3411-2225
Universidade Estadual de Feira de Santana, Brasil
E-mail: lenaldo.uefs @ gmail.com
Mauricio Santana Lordelo
ORCID: https://orcid.org/0000-0002-3791-1815
Universidade Estadual de Feira de Santana, Brasil
E-mail: mslordelo@uefs.br
Raul Pinho Sales
ORCID: https://orcid.org/0000-0003-4146-7160
Universidade Estadual de Feira de Santana, Brasil
E-mail: raulsalesagro@gmail.com

\begin{abstract}
Resumo
A Lamiaceae é uma família de plantas com importantes aptidão medicinal, entre elas, a Eplingiella fruticosa (Salzm. Ex Benth. Harley\& J.F.B. Pastore). Como a exploração desse vegetal dá-se de forma extrativista, existe um potencial risco de erosão genética, o que torna essencial estudos que incrementem estratégias de conservação, domesticação e melhoramento. O objetivo foi realizar estudos ecogeográficos com vistas a identificar interações do ambiente que possam ser preditivas do crescimento e desenvolvimento da espécie. Foram levantados dados edáficos, climáticos, relevo, geomorfológicos, fitogeográficos e biomas, nos sítios de ocorrência natural, nas mesorregiões do centro-norte, nordeste, metropolitana de Salvador na Bahia e mesorregiões leste e agreste no estado de Sergipe. As informações foram coletadas em visitas in situ e coletados em sites especializados como IBGE, INPE, INMET e EMBRAPA. Os dados foram submetidos a análise estatística (PCA). Concluiu-se que o ambiente de crescimento da espécie, tipifica-se como de solos arenosos, baixa fertilidade, elevado concentração e teor de alumínio $\left(\mathrm{Al}^{+3}\right)$, nos domínios do bioma caatinga, mata atlântica e agreste dos estados Ba e SE, em uma larga faixa de adaptação às variantes de temperatura, chuva e altitude.
\end{abstract}

Palavras-chave: Eplingiella fruticosa; Bioativos; Ecogeografia; Conservação.

\begin{abstract}
Lamiaceae is a family of vegetable species characterized by their medicinal applications, which include plants of the Eplingiella fruticosa species (Salzm. Ex Benth. Harley \& J.F.B. Pastore). This species is exploited based on extractivism; thus, genetic erosion is a potential risk, and studies focused on strategies for conservation, domestication, and breeding of this species are essential. The objective of this work was to identify interactions in the environment that may predict growth and development of this species based on ecogeographic studies. Data of soil, climate, topography, geomorphology, phytogeography, and biome were collected at naturally occurring sites in the central-north, northeast, and metropolitan regions of Salvador, state of Bahia, and in the eastern and rural mesoregions of the state of Sergipe, Brazil. Data were collected in situ and at specialized sites of the Brazilian Institute of Geography and Statistics (IBGE), Brazilian National Institute of Space Research (INPE), Brazilian National Institute of Meteorology (INMET), and Brazilian Agricultural Research Corporation (EMBRAPA) and subjected to statistical analysis (Principal Component Analysis). The growth environment of E. fruticosa plants was typified as low-fertility sandy soils with high concentration and contents of aluminum $\left(\mathrm{Al}^{+3}\right)$, within the domains of the Caatinga and Atlantic Forest biomes and Agreste region in the states of Bahia and Sergipe, with adaptation to a wide range of variation in temperature, rainfall depths, and altitude.
\end{abstract}

Keywords: Eplingiella fruticosa; Bioactive; Ecogeography; Conservation. 


\begin{abstract}
Resumen
Las lamiáceas (Lamiaceae) son una familia de plantas con importantes propiedades medicinales, entre ellas la Eplingiella fruticosa (Salzm. Ex Benth, Harley \& J.F.B. Pastore). Dado que la explotación de esta planta se realiza de manera extractiva, existe un riesgo potencial de erosión genética, lo que hace imprescindibles estudios que incrementen las estrategias de conservación, domesticación y mejoramiento. El objetivo fue realizar estudios ecogeográficos, para identificar interacciones con el medioambiente que puedan ser predictivas del crecimiento y desarrollo de la especie. Se recolectaron datos edáficos, climáticos, de relieve, geomorfológicos, fitogeográficos y de biomas, en sitios de ocurrencia natural, en las mesorregiones medio norte y noreste y en la región metropolitana de la ciudad de Salvador, en el estado de Bahía, Brasil, y en las mesorregiones este y agreste en el estado de Sergipe. La información fue recolectada en visitas in situ y de sitios web especializados, como los del Instituto Brasileño de Geografía y Estadística (IBGE), Instituto Nacional de Investigaciones Espaciales (INPE), Instituto Nacional de Meteorología (INMET) y Empresa Brasileña de Investigación Agropecuaria (Embrapa). Los datos fueron sometidos a un análisis estadístico de Análisis de Componentes Principales (en español ACP, en inglés, PCA). Se concluyó que el ambiente de crecimiento de la especie se caracteriza por ser de suelo arenoso, baja fertilidad, alta concentración y contenido de aluminio $\left(\mathrm{Al}^{+3}\right)$, en los dominios del bioma de la caatinga, la mata atlántica y del agreste de los estados de Bahía y Sergipe, en un amplio rango de adaptación a variaciones de temperatura, lluvia y altitud.
\end{abstract}

Palabras clave: Eplingiella fruticosa; Activos biológicos; Ecogeografía; Conservación.

\title{
1. Introdução
}

A região semiárida do Brasil, caracterizada por temperaturas médias anuais muito elevadas, baixa umidade, escassez e irregularidade de chuvas ao longo do ano, é constituída pelos biomas Cerrado, Caatinga e Mata Atlântica, com fitofisionomias que vão desde uma vegetação densa e úmida até caatinga (Aziz, 1999). Em função de suas condições edafoclimáticas peculiares, torna-se um dos ecossistemas mais valiosos, apresentando em seu domínio um número expressivo de espécies de plantas de elevado potencial para produção de substâncias bioativas. A síntese desses compostos ativos faz parte da complexa interação entre as plantas e as condições de estresse abiótico, típico nessa região, sobretudo de temperatura e de déficit hídrico.

Dentre as famílias botânicas típicas dessa região destaca-se a família Lamiaceae. A maioria dessas espécies são conhecidas pelo seu amplo uso e aplicação devido a presença de grande variedade de compostos estruturais (Lemes, Ferri \& Lopes, 2011). São vegetais que caracterizam-se pela presença de tricomas glandulares em suas folhas, flores e ramos, o que as tornam grandes produtoras de óleos essenciais (Silva ,2015). São compostos voláteis, constituídos de substâncias com elevada atividade biológica, como alfa e beta pineno, e-cariofilento, eugenol, timol, germacreno, entre outros, o que lhes conferem grande interesse farmacológico (Franco, Alves, Andrade, Jesus, Santos, Antoniolli \& Quintans Júnior (2011a).

O uso de plantas medicinais tem se tornado cada vez mais popular entre os povos de todo o mundo, e seu uso é cada vez mais difundido pelos profissionais de saúde. É uma prática que vem ganhando espaço na vida das pessoas em tratamentos de diversas patologias bem como melhoria na qualidade de vida (Andrade, Florêncio \& Souza, 2020).

Alecrim-de-vaqueiro (Eplingiella fruticosa (Salzm. Ex Benth. Harley \& J.F.B. Pastore), é uma Lamiaceae nativa do semiárido, com atividades biológicas comprovadas. Esta planta tem elevada importância para os povos locais que fazem o uso das folhas através da infusão, como atividade analgésica, anti-inflamatória, anticonvulsivante, tratamento de infecções na garganta e desobstrução das vias respiratórias, sendo, assim, comumente encontrada em feiras livres das localidades onde tem ocorrência (Franco et al., 2011 a). Amplas pesquisas comprovaram que o óleo essencial do alecrim-de-vaqueiro apresenta efeito vasodilatador, antinociceptivo e larvicida (Silva, Dória, Maia, Nunes, Carvalho, Blank \& Cavalcanti, 2008). Extratos de folhas dessa espécie foram usados em camundongos, e ficou constatado o efeito antinociceptivo com ausência de toxicidade aguda pelo método usado, o que sugere o uso fitoterápico desta espécie vegetal no combate a dores (Silva et al., 2008).

A espécie, apesar de apresentar ampla ocorrência na região nordeste do Brasil, em solos arenosos e pobres das áreas mais litorâneas (Silva, 2015), forma populações densas, porém com poucos indivíduos e bastante isoladas umas das outras. Adicionalmente, por ser uma espécie não cultivada, cuja exploração se dá de forma extrativista, torna-se extremamente vulnerável a erosão genética, sendo imprescindíveis estudos e ações que subsidiem trabalhos na área de conservação, 
domesticação e desenvolvimento de sistemas de cultivos (Silva, 2015). Nesse contexto, a ecogeografia é uma ferramenta de grande importância, pois permite predizer em que tipo de ambiente uma espécie nativa ocorre e, ainda, a sua adaptação aos fatores geográficos, edáficos e climáticos (Meira, 2017).

Estudos ecogeográficos têm possibilitado a caracterização dos locais de ocorrência de espécies medicinais importantes para a indústria farmacêutica, contribuindo na definição de formas de manejo da cultura, de modo a garantir as condições favoráveis ao estímulo à produção de compostos bioativos, geralmente muito afetados por fatores ambientais (Gobbo-Neto, 2007). Souza, Queiroz, Anjos, Santos, Martins, Fernandes e Costa (2008), estudando as características edafoclimáticas das áreas de ocorrência da fava d'anta (Dimorphandra mollis Benth), espécie nativa do Brasil pertencente à família Fabaceae, identificaram que os solos apresentavam baixa fertilidade, $\mathrm{pH}$ ácido e elevados teores de Alumínio $\left(\mathrm{Al}^{+3}\right)$. Oliveira, Vieira, Maia e Martins (2009), através de informações ecológicas e geográficas, caracterizaram os locais de ocorrência da poaia [Psychotria ipecacuanha (Brot) Stokes], uma planta medicinal que pertence à família Rubiácea, identificando o tipo de ambiente favorável ao cultivo da espécie. Da mesma forma, Mendes (2015), identificou as condições ecogeográficas e edafoclimáticas de ocorrência da erva baleeira (Varronia curassavica Jacq.), uma representante da família Boraginaceae.

Nesse contexto, este trabalho teve como objetivo fazer o levantamento de dados relacionados aos atributos químicos e físicos dos solos, do clima, relevo, geomorfologia, vegetação e biomas de ocorrência da espécie alecrim-de-vaqueiro, coletadas nos Estados da Bahia e Sergipe, com o objetivo de subsidiar o desenvolvimento de sistemas de cultivo e manejo da espécie.

\section{Metodologia}

O estudo tem como metodologia uma análise qualitativa e quantitativa dos fenômenos ecogeográficos que envolve a especie em dois estados de ocorrência natural. Os dados da sua localização, foram realizados no período de 2018 a 2020, com base nas informações existentes no banco de dados biológicos da Species Link (http://www.splink.org.br) bem como de fonte secundária resultado de estudos de caracterização da espécie no período de 2011 a 2015 (Silva, 2015). Foram apurados informações dos sítios de ocorrência natural de alecrim-de-vaqueiro (Eplingiella fruticosa Salzm ex Benth) Harley \& J.F.B. Pastore) nas mesorregiões centro-norte baiano, nordeste baiano e Metropolitana de Salvador no estado da Bahia e mesorregiões leste e agreste no estado de Sergipe, em um total de 22 localidades de ocorrência da espécie (figura 1). 
Tabela 1 - Código de localização, município, coordenadas geográficas, altitude, mesorregião e estado dos 22 sítios de ocorrência da espécie Alecrim-de-vaqueiro nos estados da Bahia e Sergipe. Feira de Santana, 2019.

\begin{tabular}{|c|c|c|c|c|c|}
\hline \multirow{2}{*}{ Código } & \multirow{2}{*}{ Localidade } & \multicolumn{2}{|c|}{ Coordenadas Geográficas } & \multirow{2}{*}{ Mesorregião } & \multirow{2}{*}{ Estado } \\
\hline & & Latitude & Longitude & & \\
\hline FSA & Feira de Santana & $12^{\circ} 11^{\prime} 48.8^{\prime \prime}$ & $38^{\circ} 58^{\prime} 1.66^{\prime \prime}$ & Centro norte Baiano & $\mathrm{BA}$ \\
\hline MDC I & Morro do Chapéu & $11^{\circ} 34^{\prime} 14.5^{\prime \prime}$ & $41^{\circ} 10^{\prime} 32.2$ & Centro norte Baiano & $\mathrm{BA}$ \\
\hline MDC II & Morro do Chapéu & $11^{\circ} 37^{\prime} 40.3^{\prime \prime}$ & $41^{\circ} 00^{\prime} 01.8^{\prime \prime}$ & Centro norte Baiano & BA \\
\hline MDC III & Morro do Chapéu & $11^{\circ} 35^{\prime} 53.0^{\prime \prime}$ & $41^{\circ} 09^{\prime} 49.5^{\prime \prime}$ & Centro norte Baiano & BA \\
\hline $\mathrm{JCB}$ & Jacobina & $11^{\circ} 35^{\prime} 52.3^{\prime \prime}$ & $41^{\circ} 12^{\prime} 47.7^{\prime \prime}$ & Centro norte Baiano & BA \\
\hline STE & Santa Teresinha & $12^{\circ} 41^{\prime} 02.8^{\prime \prime}$ & $39^{\circ} 35^{\prime} 03.1^{\prime \prime}$ & Centro norte Baiano & BA \\
\hline RFJ & Rafael Jambeiro & $12^{\circ} 33^{\prime} 06.8^{\prime \prime}$ & $39^{\circ} 27^{\prime} 17.3^{\prime \prime}$ & Centro norte Baiano & BA \\
\hline SGC & São Gonçalo Campos & $12^{\circ} 23^{\prime} 30.6^{\prime \prime}$ & $38^{\circ} 53^{\prime} 17.5^{\prime \prime}$ & Centro norte Baiano & BA \\
\hline UMB I & Umburanas & $10^{\circ} 23 ' 27.1^{\prime \prime}$ & $41^{\circ} 19^{\prime} 10.7^{\prime \prime}$ & Centro norte Baiano & BA \\
\hline UMB II & Umburanas & $10^{\circ} 24^{\prime} 10.6^{\prime \prime}$ & $41^{\circ} 18^{\prime} 40.3^{\prime \prime}$ & Centro norte Baiano & BA \\
\hline ESP I & Esplanada & $12^{\circ} 00 ' 23.7^{\prime \prime}$ & $38^{\circ} 15^{\prime} 53.9^{\prime \prime}$ & Nordeste Baiano & BA \\
\hline ESP II & Esplanada & $11^{\circ} 40^{\prime} 07.2^{\prime \prime}$ & $37^{\circ} 32^{\prime} 42.5^{\prime \prime}$ & Nordeste Baiano & BA \\
\hline CDE I & Conde & $11^{\circ} 38^{\prime} 75.2^{\prime \prime}$ & $37^{\circ} 32^{\prime} 56.4^{\prime \prime}$ & Nordeste Baiano & BA \\
\hline CDE II & Conde & $12^{\circ} 13^{\prime} 76.6^{\prime \prime}$ & $37^{\circ} 50^{\prime} 37.3^{\prime \prime}$ & Nordeste Baiano & BA \\
\hline ALH & Alagoinhas & $12^{\circ} 11^{\prime} 84.4^{\prime \prime}$ & $38^{\circ} 39^{\prime} 36.4^{\prime \prime}$ & Nordeste Baiano & BA \\
\hline EST & Estância & $11^{\circ} 10^{\prime} 78.8^{\prime \prime}$ & $37^{\circ} 24^{\prime} 03.9^{\prime \prime}$ & Nordeste Baiano & BA \\
\hline SBA & Saubara & $13^{\circ} 37^{\prime} 28.9^{\prime \prime}$ & $41^{\circ} 48^{\prime} 31.2^{\prime \prime}$ & Metropolitana Salvador & $\mathrm{BA}$ \\
\hline $\mathrm{SCV}$ & São Cristóvão & $10^{\circ} 55^{\prime} 33.6^{\prime \prime}$ & $37^{\circ} 11^{\prime} 56.1^{\prime \prime}$ & Leste Sergipano & SE \\
\hline JPT & Japaratuba & $10^{\circ} 35^{\prime} 54.8^{\prime \prime}$ & $36^{\circ} 59^{\prime} 17.0^{\prime \prime}$ & Leste Sergipano & $\mathrm{SE}$ \\
\hline ITD & Itaporanga D'ajuda & $11^{\circ} 09^{\prime} 04.6^{\prime \prime}$ & $37^{\circ} 24^{\prime} 10.7^{\prime \prime}$ & Leste Sergipano & $\mathrm{SE}$ \\
\hline ITB & Itabaiana & $10^{\circ} 46^{\prime} 10.4^{\prime \prime}$ & $38^{\circ} 21^{\prime} 32.0^{\prime \prime}$ & Agreste Sergipano & SE \\
\hline SMD & Simão Dias & $10^{\circ} 43^{\prime} 48.2^{\prime \prime}$ & $37^{\circ} 46^{\prime} 46.5^{\prime \prime}$ & Agreste Sergipano & SE \\
\hline
\end{tabular}

Fonte: IBGE - Instituto Brasileiro de Geografia e Estatística (2019).

A partir das coordenadas geográficas (latitude e longitude) desses locais, foram realizados levantamentos de informações climáticas de temperaturas médias anual $\left({ }^{\circ} \mathrm{C}\right)$, precipitação média anual $(\mathrm{mm})$, dados de relevo, altitude $(\mathrm{m})$, bioma, fitofisionomia e informações geomorfológicas. Arquivos vetoriais com dados de clima, geomorfologia, fitofisionomia e tipologia de solos foram obtidos dos sites do IBGE (Instituto Brasileiro de Geografia e Estatística), INMET (Instituto Nacional de Meteorologia) e EMBRAPA (Empresa Brasileira de Pesquisa Agropecuária) e, em seguida, processados e sistematizados pelo software QGIS. (Geografic Information System - GIS) versão 3.4 (madeira), Sistema de coordenadas Datum - SIRGAS 2000.Adicionalmente, foram realizadas três expedições para coleta de amostras de solo em oito sítios de ocorrência da espécie.

A localização dos sítios foi realizada com o auxílio do receptor GPS (Global Position System) Garmim eTrex@ 10, a partir dos dados das coordenadas geográficas. As coletas aconteceram nos municípios de Feira de Santana, Alagoinhas, Esplanada (sítio I e sítio II), Estância, Conde (sítio I e sítio II) e São Gonçalo dos Campos. Em cada localidade, amostras compostas foram formadas, a partir de amostras simples na comada de $0-20 \mathrm{~cm}$ de profundidade, seguindo a metodologia de Ribeiro, Gontijo \& Alvarez (1999), com auxílio de um trado holandês, totalizando 24 coletas. Todas as amostras foram retiradas na projeção do raio da copa das plantas e acondicionadas em sacos plásticos transparentes e devidamente lacrados e identificados. As análises foram realizadas no Laboratório de Análise de Fertilizantes, Solo e Monitoramento Ambiental Ltda. (LAFISMA), localizada na cidade de Cruz das Almas-Ba. Foram quantificadas as propriedades químicas $\left(\mathrm{pH}, \mathrm{P}, \mathrm{K}^{+}, \mathrm{Ca}^{+2}\right.$, 
$\mathrm{Mg}^{+2}, \mathrm{Al}+\mathrm{H}, \mathrm{M} . \mathrm{O}$ ), soma das bases (SB), saturação de base (V \%), saturação de alumínio (m \%), CTC e físicas (textura e granulométrica). As concentrações químicas e análise física foram determinadas de acordo com o preconizado por Teixeira, Donagemma \& Fontana (2017). Os solos foram caracterizados seguindo a classificação proposta pelo Sistema Brasileiro de Classificação de Solos (SiCBS - Sistema Brasileiro de Classificação de Solos; EMBRAPA, 2018). Os dados das análises químicas foram submetidos à análise de componentes principais (PCA). Inicialmente as variáveis foram submetidas à análise de correlação de Pearson ( $\mathrm{r}$ ( $\mathrm{p}<=0,5)$, para avaliar a intensidade da correlação existente que justificasse seu uso na matriz da análise dos dados. A retenção dos eixos da PCA interpretados, foi pela redução do conjunto de dados em combinações lineares, com escores gerados em torno de 80 \% da variação total dos dados (Cruz \& Souza, 2006). Esse modelo, permitiu identificar propriedades químicas mais relevantes em seus diferentes habitats de ocorrência, favorecendo identificação de grupos com base em suas medidas e similaridades. Essa análise foi processada com o auxílio do software R 3.6.0 (R Development Core Team, 2019).

\section{Resultados e Discussão}

Os 22 sítios de ocorrência amostrados estão compreendidos entre as coordenadas geográficas com latitude de $10^{\circ} 24^{\prime}$

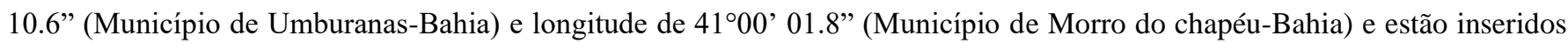
nos biomas Caatinga, Mata Atlântica e uma zona de transição entre Mata Atlântica e a Caatinga, a sub região, o Agreste (Tabela 2). A Mata Atlântica é o domínio em que estão inseridos $41 \%$ dos sítios - ALH, CDE I, CDE II, ESP I, ESP II, EST, JPT, SBA e SCV; seguidos da Caatinga (41\%) - JCB, MDC I, MDC II, MDC II, STE, UMB I, UMB II, SMD, e ITB e zona de transição (18\%) - FSA, RFJ, SGC e ITD. Esses dados demonstram que a espécie possui uma acentuada plasticidade fenotípica, caracterizada por uma larga faixa de tolerância a ecossistemas diferentes. As espécies vegetais podem apresentar um intervalo de tolerância variável para diferentes fatores do ambiente em que estão inseridas e que são resultantes da atuação continua dos fatores evolutivos (Ross,2014). As plantas da família Lamiaceae têm ocorrência ampla e estão presentes em vários ecossistemas e ocorrem praticamente em todos os continentes e nos mais diversos ambientes (Harley 2014; Harley, 2012). No Brasil, populações nativas da espécie em estudo foram identificadas desde o Ceará até o Estado de Minas Gerais (Harley, 2014). Esses estudos confirmam a vocação da família na adaptação aos vários ambientes, que vão desde um domínio vegetal caracterizado por uma floresta densa e de acentuada umidade até uma região de caatinga, descrita por matas secas e abertas.

A maioria dos sítios de ocorrência da espécie está inserido em um espaço geográfico tipo restinga. Esse tipo de vegetação abrange doze (12) sítios de coleta, localizados nas cidades de Alagoinhas (ALH), Conde (CDE I e II), Esplanada (ESP I e II), Estancia (EST), Saubara (SBA) e São Gonçalo dos Campos (SGC) na Bahia e, Itabaiana (ITB), Itaporanga D’ajuda (ITD), Japaratuba (JPT), e São Cristóvão (SCV) no estado de Sergipe (Tabela 2). As restingas são formações situadas em paralelo a Zona Costeira e são caracterizadas por possuir uma vegetação arbustiva, areias soltas e nuas, sendo pobres em nutrientes e com baixa capacidade de retenção de água nas camadas mais superficiais do perfil, de um solo predominantemente arenoso. É um espaço geográfico de elevadas temperaturas, tanto na atmosfera como nas camadas superficiais do solo. Sofrem, com razoável frequência, influência marinha e pressões antropogênicas, tais como, expansão das áreas urbanas, construções de vias e estradas. Esse cenário corrobora com o trabalho de Silva (2015), que identificou que a espécie alecrim-de-vaqueiro ocorre no semiárido do nordeste brasileiro, em solos arenosos empobrecidos, nos estados da Bahia, Sergipe, Pernambuco, Paraíba, Ceará e Rio Grande do Norte, em solos de textura arenosa de caatinga, tabuleiros e restingas, principalmente à margem das estradas e em locais antropizados.

Apesar da predominância de sítios de ocorrência em ambientes de restinga, alguns sítios encontram-se afastados das zonas costeiras, como JCB, MDC I, MDC II, MDC III, UMB I, UMB II, na Bahia e SMD no estado de Sergipe. Esse cenário configura uma distribuição esparsa da espécie, que para Ross (2014), é possível em virtude das espécies, muitas vezes, 
habitarem áreas onde as condições não são ótimas, mas estão sempre dentro do seu intervalo de tolerância, sendo possível encontrar para uma mesma espécie diferentes biótipos por conta dessa tolerância. A grande maioria (68\%) das populações foi encontrada em localidades cuja temperatura anual média encontra-se na faixa entre $24^{\circ} \mathrm{C}$ e $25^{\circ} \mathrm{C}$ e inseridas em um território em que o plano de relevo vai desde o nível do mar até 460 metros de altitude. Destacam-se aqui as localidades MCD I, MCD II, MCD III e JCB, UMB I e UMB II, que apresentaram temperaturas médias variando entre $20^{\circ} \mathrm{C}$ e $22^{\circ} \mathrm{C}$, atípicas para o bioma Caatinga, em que estão inseridas. Esse fato está relacionado às características topográficas da região, que é marcada por cadeias montanhosas, predominância de planaltos e serras, tipo climático tropical de altitude, altitude elevadas, podendo passar dos 1.000 m, a exemplo da cidade de Morro do Chapéu, na região da Chapada Diamantina na Bahia.

As séries pluviométricas revelaram que $41 \%$ das localidades de ocorrência naturais de alecrim-de-vaqueiro estão situadas em uma faixa de precipitação média de até $800 \mathrm{~mm}$ anuais, $14 \%$ entre $800 \mathrm{~mm}$ até $1200 \mathrm{~mm}$ anuais e $45 \%$ acima dos $1200 \mathrm{~mm}$ anuais (Tabela 2). Os extremos pluviométricos estão nos municípios de Umburanas-Bahia e Conde-Bahia com 553 $\mathrm{mm}$ e $1796 \mathrm{~mm}$ de chuvas respectivamente.

Tabela 2 - Código localização, municípios, clima, bioma, temperatura. Índice pluviométrico e geomorfologia das 22 localidades de ocorrência alecrim-de-vaqueiro (Eplingiella fruticosa (Salzm ex Benth.) Harley \& J.F.B. Pastore) coletadas nos estados da Bahia e Sergipe. Feira de Santana, 2019.

\begin{tabular}{|c|c|c|c|c|c|c|}
\hline Localidade & Tipologia Climática & Bioma & $\begin{array}{l}\text { Temperatura } \\
\left({ }^{\circ} \mathrm{C}\right)\end{array}$ & $\begin{array}{l}\text { Chuva } \\
(\mathrm{mm})\end{array}$ & Geomorfologia & $\begin{array}{c}\text { Altitude } \\
(\mathrm{m})\end{array}$ \\
\hline FSA & Tropical seco úmido & Caatinga/Agreste & 24,00 & 754 & Depressão & 300 \\
\hline MDC I & Tropical seco úmido & Caatinga & 20,00 & 691 & Serras & 1094 \\
\hline MDC II & Tropical seco úmido & Caatinga & 20,00 & 691 & Serras & 1011 \\
\hline MDC III & Tropical seco úmido & Caatinga & 20,00 & 691 & Serras & 1015 \\
\hline $\mathrm{JCB}$ & Tropical seco & Caatinga & 24,00 & 831 & Serras & 461 \\
\hline STE & Tropical seco & Caatinga & 23,00 & 651 & Depressão & 236 \\
\hline RFJ & Tropical seco & Caatinga/Agreste & 23,00 & 647 & Depressão & 220 \\
\hline $\mathrm{SCV}$ & Litorâneo úmido & Mata Atlântica & 25,00 & 1.372 & Tabuleiros & 37 \\
\hline JPT & Litorâneo úmido & Mata Atlântica & 25,00 & 1.343 & Tabuleiros & 112 \\
\hline ESP I & Litorâneo úmido & Mata Atlântica & 24,00 & 1.226 & Tabuleiros & 160 \\
\hline ESP II & Litorâneo úmido & Mata Atlântica & 24,00 & 1.226 & Tabuleiros & 126 \\
\hline ITB & Tropical Seco & Caatinga & 24,00 & 1.044 & Tabuleiros & 239 \\
\hline SBA & Litorâneo úmido & Mata Atlântica & 24,00 & 1.680 & Tabuleiros & 16 \\
\hline SGC & Tropical seco úmido & Caatinga/Agreste & 24,00 & 1.079 & Tabuleiros & 219 \\
\hline UMB I & Tropical seco & Caatinga & 22,00 & 553 & Serras & 609 \\
\hline UMB II & Tropical seco & Caatinga & 22,00 & 553 & Serras & 609 \\
\hline CDE I & Litorâneo úmido & Mata Atlântica & 25,00 & 1.796 & Tabuleiros & 12 \\
\hline CDE II & Litorâneo úmido & Mata Atlântica & 25,00 & 1.796 & Tabuleiros & 12 \\
\hline ALH & Litorâneo úmido & Mata Atlântica & 24,00 & 1.478 & Tabuleiros & 50 \\
\hline EST & Litorâneo úmido & Mata Atlântica & 25,00 & 1.465 & Tabuleiros & 44 \\
\hline ITD & Tropical úmido & Caatinga/Agreste & 25,00 & 1.395 & Tabuleiros & 9 \\
\hline SMD & Tropical Seco & Caatinga & 24,00 & 917 & Planície & 250 \\
\hline
\end{tabular}

Fonte: IBGE - Instituto Brasileiro de Geografia e Estatística (2019); INMET - Instituto Nacional de Meteorologia (2019).

Os dados sugerem que, além dos fatores do bioma, tipografia climática, altitude e geomorfologia, também a pluviometria se mostra como um fator não determinante para o estabelecimento da espécie, o que pode justificar seu amplo intervalo de tolerância e adaptação aos diversos fatores ambientais, favorecendo assim, uma diversidade de ecótipos e ampla faixa nos limites naturais de distribuição. Essa generalidade de ocorrência de ambientes, também foi observada por Meira (2017), em um estudo realizado com Lippia rotundifolia Cham, espécie vegetal de aplicação medicinal, com registros em quatro meso regiões no estado de Minas Gerais.

$\mathrm{Na}$ análise física do solo verificou-se a granulometria com textura média (\% de argila entre 15 e $35 \%$ ) em 92\% dos solos amostrados, e solos arenosos (teor de argila de até $15 \%$ ) em apenas $8 \%$ em CDE sitio I e CDE sitio II , (Figura 1) Estes 
resultados estão de acordo com o observado por Silva (2015), quando relatou que a espécie ocorre no semiárido do nordeste brasileiro, em solos de graduação textural arenosa da caatinga, tabuleiros e restingas, principalmente os situados às margens de estradas.

Figura 1 - Caracterização da textura do solo em 8 locais de ocorrência natural de alecrim-de-vaqueiro (Eplingiella. Fruticosa (Salzm ex Benth.) Harley \& J.F.B. Pastore. Feira de Santana, 2019.

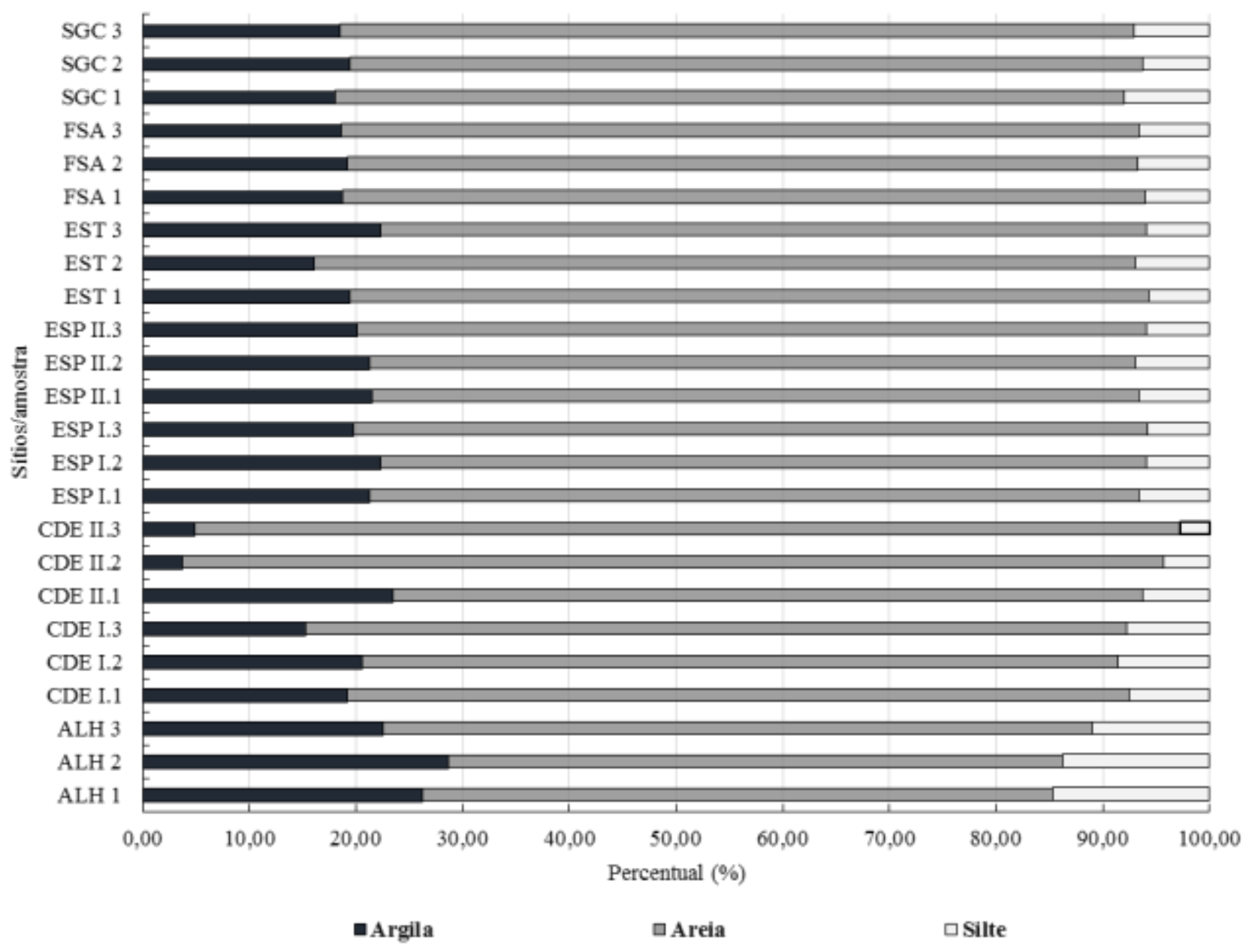

Fonte: Autores.

Nos locais de ocorrência da espécie foi observado agrupamento de indivíduos formando populações pequenas, porém densas, e isoladas das demais, se diferenciando e destacando das formações vegetais do entorno da área. Esse fato pode ser decorrente das características físicas do solo, por apresentar em seu perfil pouca retenção de água, conferindo-lhe uma maior permeabilidade e uma consequente perda de umidade e lixiviação dos nutrientes, tornando-o um substrato pobre, de baixa fertilidade e, quase sempre, inapropriado para espécies mais exigentes de culturas domesticadas. Esse mesmo cenário foi verificado por Magnago, Martins, Schaefer e Neri (2010), em estudo de caracterização fitofisionômica e suas correlações com os atributos pedológicas de um solo no estado de Minas Gerais, onde o solo foi um dos fatores determinantes da distribuição da vegetação, associado a disponibilidade de água e a interferência de luz.

A maioria dos sítios de ocorrência da espécie está localizado em Neossolos litólicos (41\%) (MDC I, MDC II, MDC III, JPT, SBA, UMB I, UMB II, ALH e SMD) e Argissolos Vermelho-Amarelo (41\% dos sítios) (FSA, ESP I, ESP II, SGC, ITD, SCV, EST, CDE I e CDE II). Os demais (14\%) foram encontrados em Chernossolo argilúvico, Neossolos Quartzênico e Planossolo álicos. Predominantemente, esses solos estão inseridos em um ambiente fitogeográfico caracterizados como 
Caatinga - $55 \%$ (FSA, MDC I, MBC II, MDC III, STE, ITB, SGC, UMB I, UMB II, EST e SMD), além de Floresta do Interior (27 \%) (SCV, JPT, ESP I, ESP II, ALH I, ITD), Floresta Costeira (14 \%) (SBA, CDE I, e CDE II) e Floresta Atlântica Seca (5\%) (JCB) (Tabela 3).Os Neossolos litólicos estão localizadas em heterogêneos domínios geomorfológicos, que vão desde as Serras da Chapada Diamantina (MDC I, MDC II, MDC III, UMB I, UMB II), Tabuleiros do Recôncavo (ALH), Tabuleiro Costeiro (JPT), Tabuleiro do Rio Vaza Barris (SMD) e Planície (SBA). São geralmente solos rasos e pedregosos, com contato lítico entre 50 e $60 \mathrm{~cm}$ de profundidade, pequena capacidade de armazenamento de água e alta suscetibilidade a erosão. Estas características conferem a esses solos limitações ao uso agrícola (Dos Santos, Jacomine, dos Anjos, De Oliveira, Lumbreras, Coelho \& Cunha, 2018). Os Argissolos estão localizados em relevos de depressões do Paraguaçu (FSA e SGC) e em Tabuleiros Costeiros (ITD, EST, ESP, CDE I e CDE II) (Tabela 3). São solos minerais bastante intemperizados, bem desenvolvidos e drenados, com boa capacidade de armazenamento de água e, de modo geral, como fator limitante uma baixa fertilidade natural, mas que não lhe tira a aptidão agrícola (Dos Santos et al., 2018).

Tabela 3 - Código das localidades, classificação de solo, fitofisiografia e geomorfologia de sítios de ocorrência de Alecrim de vaqueiro (Eplingiella fruticosa) (Salzm ex Benth.) Harley \& J.F.B. Pastore) nos estados da Bahia e Sergipe. Feira de Santana, 2019.

\begin{tabular}{|c|c|c|c|}
\hline $\begin{array}{l}\text { Código da } \\
\text { Localidade }\end{array}$ & Classificação do Solo & Fitogeografia & Geomorfologia \\
\hline ALH & Neossolos Litólicos & Floresta do Interior & Tabuleiros do Recôncavo \\
\hline CDE I & Argissolo Vermelho-Amarelo & Floresta Costeira & Planícies Marinhas \\
\hline CDE II & Argissolo Vermelho-Amarelo & Floresta Costeira & Planícies Marinhas \\
\hline ESP I & Argissolo Vermelho-Amarelo & Floresta do Interior & Tabuleiros do Recôncavo/Tucano \\
\hline ESP II & Argissolo Vermelho-Amarelo & Floresta do Interior & Tabuleiros do Recôncavo/Tucano \\
\hline EST & Argissolo Vermelho-Amarelo & Caatinga & Tabuleiros Costeiros \\
\hline FSA & Argissolo Vermelho-Amarelo & Caatinga & Depressão dos Rio Paraguaçu \\
\hline ITB & Neossolos Quartzênico & Caatinga & Tabuleiros do Recôncavo \\
\hline ITD & Argissolo Vermelho-Amarelo & Floresta do Interior & Tabuleiros Costeiros \\
\hline MDC I & Neossolos Litólicos & Caatinga & Serras da Diamantina \\
\hline JPT & Neossolos Litólicos & Floresta do Interior & Tabuleiros Costeiros \\
\hline MDC II & Neossolos Litólicos & Caatinga & Serras da Diamantina \\
\hline $\mathrm{JCB}$ & Latossolo Amarelo & Floresta Atlântica Seca & Serras da Diamantina \\
\hline MDC III & Neossolos Litólicos & Caatinga & Serras da Diamantina \\
\hline RFJ & Chernossolo Argilúvico & Caatinga & Depressão dos Rios Paraguaçu \\
\hline SBA & Neossolos Litólicos & Floresta costeira & Planície Marinhas \\
\hline $\mathrm{SCV}$ & Argissolo Vermelho-Amarelo & Floresta do Interior & Tabuleiros Costeiros \\
\hline SGC & Argissolo Vermelho-Amarelo & Caatinga & Depressão dos Rio Paraguaçu \\
\hline SMD & Neossolos Litólicos & Caatinga & Tabuleiros do Rios Real \\
\hline STE & Planossolo Háplico & Caatinga & Depressão dos Rio Paraguaçu \\
\hline UMB I & Neossolos Litólicos & Caatinga & Serras da Diamantina \\
\hline UMB II & Neossolos Litólicos & Caatinga & Serras da Diamantina \\
\hline
\end{tabular}

Fonte: IBGE (2019); EMBRAPA (2018).

Os dados dos atributos químicos do solo, em quase sua totalidade (98\%), demonstram que os sítios de ocorrência da espécie estão em solos distróficos, com as bases $\left(\mathrm{Ca}^{+2}, \mathrm{Mg}^{+2}, \mathrm{~K}^{+}\right)$ocupando menos que $50 \%$ da CTC dos solos (Tabela 4). A saturação de alumínio foi considerada muito baixa $(\mathrm{m} \%<=15)$ em $16 \%$ das amostras analisadas, enquanto que $54 \%$ apresentaram saturação alta a muito alta (m\% >=35), Ribeiro et al. (1999). O mesmo autor afirma ainda, que essa condição edáfica está associada ao caráter ácido de um solo e favorece a presença de elevados teores de $\mathrm{Al}^{+3}$ no complexo de troca catiônica. Nesse sentido, nas localidades analisadas, $88 \%$ do substrato de desenvolvimento do alecrim de vaqueiro apresentouse com caráter ácido $(\mathrm{pH}<=5,4)$ e, com mais da metade das amostras $(58 \%)$ com concentrações de $\mathrm{Al}^{+3}$ enquadradas como média a muito alta $\left(\mathrm{Al}^{+3}>=0,20 \mathrm{cmol}_{\mathrm{c}} \cdot \mathrm{dm}^{-3} \cdot 20 \mathrm{cmol}_{\mathrm{c}} \cdot \mathrm{dm}^{-3}\right.$. Foram observados ainda que em $88 \%$ dos sítios de ocorrência, os valores de saturação de bases enquadraram-se como muito baixo a baixo (V\% <= 40). Esses resultados demonstram que a 
espécie ocorre predominantemente em solos com pequena quantidade de cátions considerados essenciais aos vegetais e, solos com seus coloides saturados predominantemente por $\left(\mathrm{H}^{+}+\mathrm{Al}^{+3}\right)$, cátions potencialmente tóxicos e componentes da acidez.

Tabela 4 - Atributos dos solos em oito localidades de ocorrência natural de alecrim-de-vaqueiro [Eplingiella fruticosa (Salzm Benth.)]. Feira de Santana, 2019.

\begin{tabular}{|c|c|c|c|c|c|c|c|c|c|c|c|c|c|c|c|c|}
\hline \multirow{3}{*}{ Local } & \multirow{3}{*}{$\begin{array}{l}\text { Amo } \\
\text { stra }\end{array}$} & \multicolumn{15}{|c|}{ Atributos do Solo } \\
\hline & & \multirow{2}{*}{$\mathrm{pH}$} & $\mathrm{P}$ & $\mathrm{K}^{+}$ & $\mathrm{Ca}^{+2}$ & $\mathrm{Mg}^{+2}$ & $\mathrm{Al}^{+3}$ & $\mathrm{H}+\mathrm{Al}$ & SB & CTC & $\mathrm{V}$ & $\mathrm{m}$ & M.O & Argila & Areia & Silte \\
\hline & & & \multicolumn{2}{|c|}{ mg.dm ${ }^{-3}$} & \multicolumn{6}{|c|}{$\mathrm{cmol}_{\mathrm{c} . \mathrm{dm}^{-3}}$} & \multicolumn{6}{|c|}{$\%$} \\
\hline \multirow{3}{*}{ ALH } & 1 & 4,26 & 2,00 & 17,00 & 0,50 & 0,50 & 0,10 & 2,86 & 1,08 & 3,94 & 27,41 & 8,47 & 1,11 & 26,26 & 59,03 & 14,71 \\
\hline & 2 & 3,88 & 3,00 & 28,00 & 0,20 & 0,20 & 0,20 & 3,50 & 0,52 & 4,02 & 12,93 & 27,77 & 1,02 & 28,70 & 57,50 & 13,80 \\
\hline & 3 & 3,70 & 3,00 & 30,00 & 0,20 & 0,20 & 0,20 & 3,34 & 0,50 & 3,84 & 13,02 & 28,57 & 1,12 & 22,60 & 66,40 & 11,00 \\
\hline \multirow{3}{*}{ CDE I } & 1 & 4,00 & 3,00 & 24,00 & 0,60 & 0,40 & 0,30 & 3,47 & 1,10 & 4,57 & 24,07 & 21,42 & 0,90 & 19,20 & 73,30 & 7,50 \\
\hline & 2 & 3,86 & 3,00 & 33,00 & 0,60 & 0,50 & 0,40 & 3,65 & 1,22 & 4,87 & 25,05 & 24,69 & 1,02 & 20,60 & 70,80 & 8,60 \\
\hline & 3 & 4,07 & 4,00 & 36,00 & 0,30 & 0,30 & 0,40 & 3,76 & 0,75 & 4,51 & 16,62 & 34,78 & 0,96 & 15,30 & 76,90 & 7,80 \\
\hline \multirow{3}{*}{ CDE II } & 1 & 4,10 & 5,00 & 38,00 & 0,50 & 0,30 & 0,20 & 3,85 & 0,93 & 5,13 & 18,12 & 17,69 & 0,11 & 23,50 & 70,20 & 6,30 \\
\hline & 2 & 4,20 & 5,00 & 29,00 & 0,20 & 0,20 & 0,20 & 3,89 & 0,49 & 4,38 & 11,18 & 28,98 & 0,92 & 3,70 & 92,00 & 4,30 \\
\hline & 3 & 3,88 & 3,00 & 32,00 & 0,20 & 0,10 & 0,40 & 3,96 & 0,50 & 4,46 & 11,21 & 44,44 & 0,94 & 4,90 & 92,30 & 2,80 \\
\hline \multirow{3}{*}{ ESP I } & 1 & 4,63 & 2,00 & 24,00 & 0,20 & 0,20 & 0,20 & 4,10 & 0,50 & 4,60 & 10,86 & 28,57 & 1,08 & 21,28 & 72,12 & 6,60 \\
\hline & 2 & 3,93 & 3,00 & 26,00 & 0,20 & 0,20 & 0,30 & 4,26 & 0,38 & 4,64 & 8,18 & 44,11 & 1,23 & 22,31 & 71,82 & 5,87 \\
\hline & 3 & 4,20 & 5,00 & 36,00 & 0,70 & 0,30 & 0,20 & 3,50 & 1,11 & 4,61 & 24,07 & 15,26 & 1,07 & 19,79 & 74,36 & 5,84 \\
\hline \multirow{3}{*}{ ESP II } & 1 & 5,60 & 3,00 & 21,00 & 1,50 & 1,00 & 0,05 & 2,47 & 2,60 & 5,07 & 52,00 & 1,88 & 0,12 & 21,49 & 71,85 & 6,66 \\
\hline & 2 & 3,90 & 3,00 & 28,67 & 1,20 & 0,80 & 0,05 & 2,36 & 2,11 & 4,47 & 47,20 & 2,31 & 1,13 & 21,32 & 71,78 & 6,90 \\
\hline & 3 & 4,00 & 3,00 & 34,00 & 0,90 & 0,50 & 1,80 & 4,70 & 1,51 & 6,21 & 24,31 & 54,38 & 1,11 & 20,18 & 73,86 & 5,96 \\
\hline \multirow{3}{*}{ EST } & 1 & 3,80 & 3,00 & 25,67 & 0,50 & 0,50 & 2,00 & 5,67 & 1,10 & 6,77 & 16,24 & 64,51 & 1,00 & 19,48 & 74,81 & 5,71 \\
\hline & 2 & 3,70 & 3,00 & 27,00 & 0,80 & 0,40 & 0,90 & 4,00 & 1,38 & 5,38 & 25,65 & 39,47 & 0,09 & 16,10 & 76,90 & 7,00 \\
\hline & 3 & 3,25 & 3,00 & 24,00 & 0,50 & 0,50 & 1,90 & 4,84 & 1,16 & 6,00 & 19,33 & 62,09 & 0,12 & 22,31 & 71,82 & 5,87 \\
\hline \multirow{3}{*}{ FSA } & 1 & 3,87 & 5,00 & 36,00 & 0,70 & 0,50 & 2,10 & 5,36 & 1,33 & 6,69 & 19,88 & 61,22 & 0,12 & 18,80 & 75,20 & 6,00 \\
\hline & 2 & 4,50 & 9,00 & 43,00 & 1,50 & 0,50 & 0,10 & 2,45 & 2,15 & 4,60 & 46,73 & 4,44 & 1,13 & 19,20 & 74,10 & 6,70 \\
\hline & 3 & 4,10 & 12,00 & 50,00 & 0,80 & 0,40 & 0,30 & 2,76 & 1,35 & 4,11 & 32,84 & 18,18 & 1,26 & 18,60 & 74,80 & 6,60 \\
\hline \multirow{3}{*}{ SGC } & 1 & 3,98 & 5,00 & 36,00 & 0,50 & 0,50 & 1,00 & 3,78 & 1,14 & 4,92 & 23,17 & 46,72 & 1,13 & 18,10 & 73,90 & 8,00 \\
\hline & 2 & 4,56 & 5,00 & 34,00 & 0,80 & 0,50 & 0,40 & 2,84 & 1,42 & 4,26 & 33,33 & 21,97 & 1,11 & 19,40 & 74,30 & 6,30 \\
\hline & 3 & 4,63 & 6,00 & 38,00 & 0,70 & 0,30 & 0,30 & 3,26 & 1,19 & 4,45 & 26,74 & 20,13 & 1,20 & 18,50 & 74,46 & 7,04 \\
\hline
\end{tabular}

Fonte: LAFSMA - Laboratório de Fertilizantes, solos e Monitoramento ambiental Ltda.

Oliveira et. al (2009), também identificaram em locais de ocorrência de poaia [P. ipecacuanha (Brot) Stokes], espécie da família Rubiácea, que $89 \%$ das populações amostradas se desenvolveram em solos com saturação de bases menor que 50\%, portanto, solos distróficos, e destes, $59 \%$ foram considerados álicos $\left(\mathrm{m} \%>=50 \mathrm{e} \mathrm{Al}^{+3}>=0,3 \mathrm{cmol}_{\mathrm{c}}\right.$ ), ou seja, solos de atributos similares ao encontrado neste estudo, com fertilidade muito baixa e teores de alumínio acima do tolerado por um grande número de culturas convencionais. Um comportamento similar também foi observado por Melo (2012), trabalhando com dados ecológicos e geográficos de Lippia sidoides (Verbenaceae), uma importante espécie medicinal, onde constatou a presença e distribuição dessa espécie em locais caracterizados por apresentar uma vegetação do tipo caatinga e um clima semiárido, marcado por possuir atributos de solos pobres e acidez elevada, fatores similares ao encontrado neste estudo nos vários ecossistemas de ocorrência natural de alecrim-de-vaqueiro.

Análise dos Componentes Principais (PCA) dos atributos dos solos demonstrou que dois componentes, CP1 e CP2, são responsáveis por explicar 79,10 \% da variância total dos resultados amostrais coletados nos ambientes de localização da espécie em estudo (Tabela 5). O primeiro componente principal (CP1) explicou 48,5\% da variância, com as cargas fatoriais variando de 0,02 a 0,41(Tabela 5), sendo que os maiores escores foram atribuídos aos atributos de Saturação de Alumínio $(\mathrm{m} \%)$, concentração de Alumínio $\left(\mathrm{Al}^{+3}\right)$ e, como fator de contraste positivo, a matéria orgânica (M.O), podendo, por isso, denominar a CP1 como a componente do teor de $\mathrm{Al}^{+3}$, saturação de alumínio (m\%) e matéria orgânica (M.O) (Figura $2 \mathrm{~A}$ ). 
Tabela 1 - Coeficientes de ponderação dos atributos químicos do solo e a correlação com as componentes principais 1 e 2 dos fatores edáficos dos sítios de ocorrência de Eplingiella. Fruticosa (Salzm ex Benth.) Harley \& J.F.B. Pastore. Feira de Santana, Ba. 2019.

\begin{tabular}{|c|c|c|}
\hline \multirow{2}{*}{ Componentes da Variância } & \multicolumn{2}{|c|}{ Componente Principal } \\
\hline & $\mathrm{CP} 1$ & $\mathrm{CP} 2$ \\
\hline Variabilidade (\%) & 0,485 & 0,305 \\
\hline Variabilidade acumulada (\%) & 0,485 & 0,795 \\
\hline Variáveis & \multicolumn{2}{|c|}{ Carga do Fator } \\
\hline $\mathrm{pH}$ & 0,24 & 0,28 \\
\hline $\mathrm{P}$ & $-0,26$ & $-0,11$ \\
\hline K & $-0,22$ & $-0,07$ \\
\hline $\mathrm{Ca}$ & $-0,09$ & 0,53 \\
\hline $\mathrm{Mg}$ & $-0,11$ & 0,50 \\
\hline $\mathrm{Al}$ & $-0,41$ & 0,09 \\
\hline $\mathrm{H}+\mathrm{Al}$ & $-0,39$ & $-0,14$ \\
\hline $\mathrm{T}(\mathrm{CTC})$ & $-0,40$ & 0,15 \\
\hline $\mathrm{V}(\%)$ & 0,02 & 0,53 \\
\hline M.O & 0,36 & $-0,06$ \\
\hline $\mathrm{m}(\%)$ & $-0,40$ & $-0,10$ \\
\hline
\end{tabular}

$\mathrm{CP} 1=0,24 \mathrm{pH}+0,36 \mathrm{M} . \mathrm{O}+0,02 \mathrm{~V}(\%)-0,26 \mathrm{P}-0,22 \mathrm{~K}-0,09 \mathrm{Ca}-0,11 \mathrm{Mg}-0,41 \mathrm{Al}-0,39(\mathrm{H}+\mathrm{Al})-0,40 \mathrm{~T}(\mathrm{CTC})-0,40 \mathrm{~m}(\%)$ $\mathrm{CP} 2=0,28 \mathrm{pH}+0,53 \mathrm{Ca}+0,50 \mathrm{Mg}+0,53 \mathrm{~V}(\%)+0,15 \mathrm{~T}(\mathrm{CTC})+0,09 \mathrm{Al}-0,11 \mathrm{P}-0,07 \mathrm{~K}-0,14(\mathrm{H}+\mathrm{Al})-0,06 \mathrm{M} . \mathrm{O}-0,10 \mathrm{~m}(\%)$. Fonte: Autores.

Nesse componente, destacam-se os sítios de FSA e EST, com as maiores saturação de m (\%) e $\mathrm{Al}^{+3}$ presente (Figura 2 B). Ainda com base nesse eixo (CP1), pode-se inferir também, que as localidades de ALH, ESP, CDE I e SGC apresentam maiores teores de M.O e, como contraste, os menores teores de saturação de ( $\mathrm{m} \%$ ) e concentração $\mathrm{Al}^{+3}$.O segundo componente

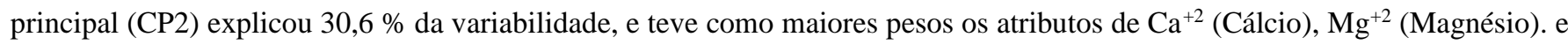
V\% (Saturação de Bases), com os escores variando de 0,06 a 0,53, sendo assim denominado de componente das bases e saturação de bases.

Figura 2 - Biplot das componentes principais 1 e 2 dos atributos químicos do solo em 8 localidades de ocorrência natural de alecrim de vaqueiro.
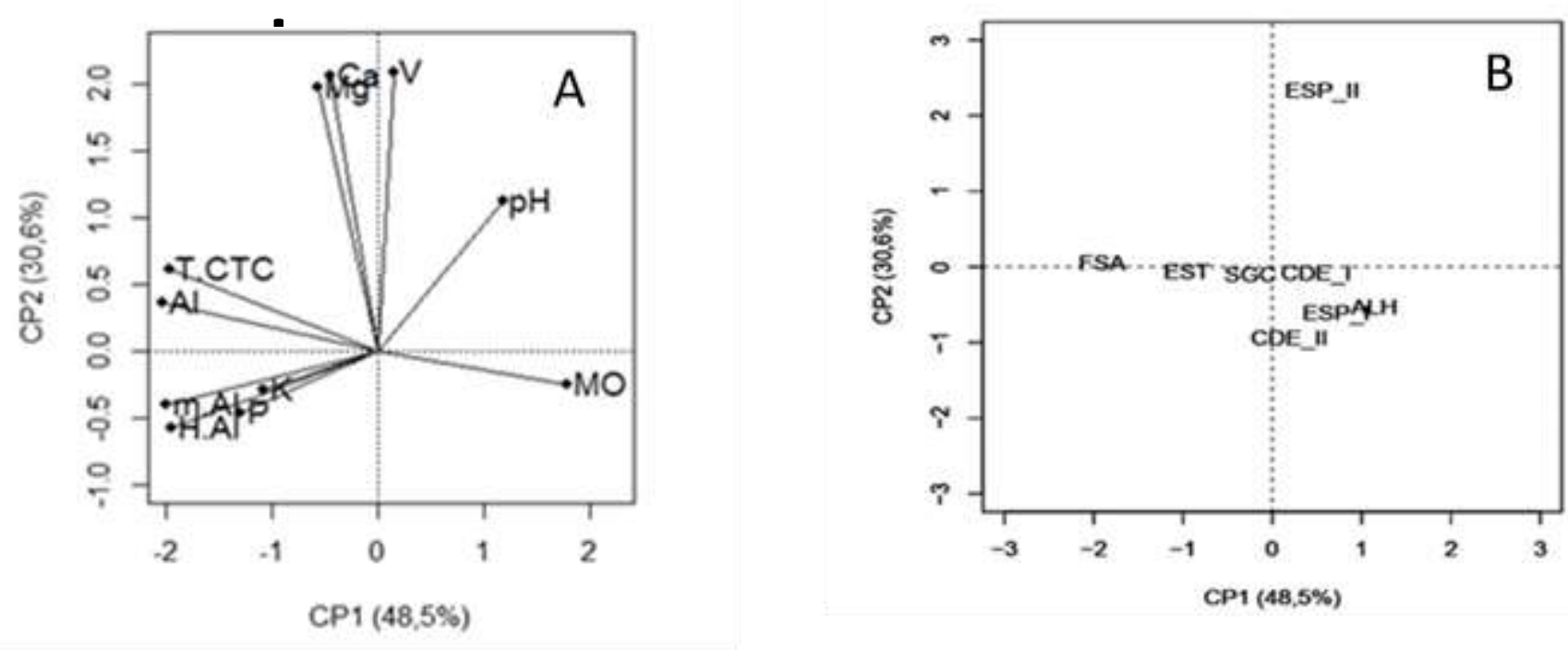

Fonte: Autores. 


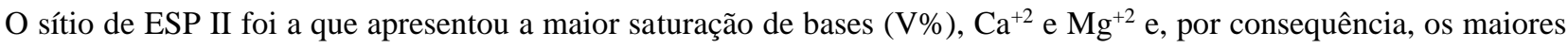
valores de Soma das Bases (SB). O local com menores teores desses atributos $\left[\mathrm{Ca}^{+2}, \mathrm{Mg}^{+2}\right.$ e V (\%)], foi o sítio CDE II, que está posicionado na parte inferior do plano (Figura 2 B).

De acordo com o perfil edafológico dos sítios de ocorrência da espécie, pode-se formar 03 agrupamentos (Figura 3), com similaridades nos perfis de fertilidade do solo. No grupo 1, caracterizado por apresentar maiores teores de M.O em relação aos demais pontos, estão os sítios de ALH, CDE I, ESP I, SGC e CDE.O grupo 2, formado por FSA e EST possui, comparado com as demais localidades de coleta, as maiores concentrações de $\mathrm{Al}^{+3}$ e saturação de $\mathrm{Al}$ (m\%). E, por fim, posicionado de forma isolada, está a localidade de ESP II, compondo o grupo 3, com o perfil edáfico marcado com concentrações de $\mathrm{Ca}^{+2}$, $\mathrm{Mg}^{+2} \mathrm{e} \mathrm{V}(\%)$ acentuadas, comparados com os demais grupos.

Figura 3 - Composição dos grupos com similaridade de locais e atributos de fertilidade de solos nas localidades de ocorrência de alecrim-de-vaqueiro. Feira de Santana, 2019.

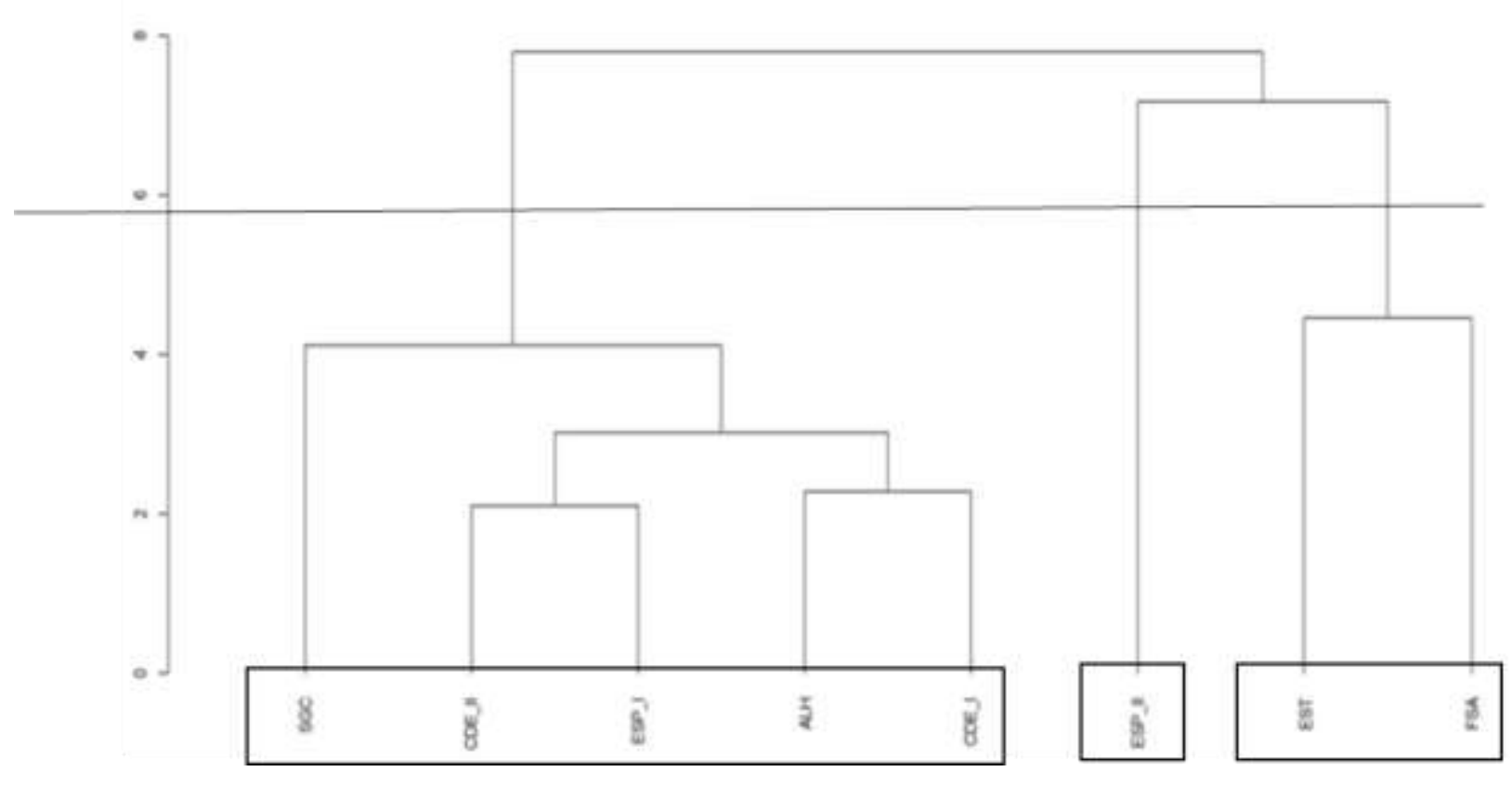

Fonte: Autores.

Com base no exposto, verifica-se que as localidades onde foram encontradas a espécie possuem atributos edáficos muito abrangentes, mas com tendência a atributos característicos de solo arenosos, uma baixa retenção de nutrientes no complexo de troca catiônica, com perfil vulnerável à erosão e de pouca aptidão agrícola, também observados por Silva (2015).

\section{Conclusão}

Conclui-se que as populações de alecrim-de-vaqueiro nas localidades estudadas, possuem ampla plasticidade em relação aos fatores ecogeográficos, o que favorece sua adaptação aos diferentes ecossistemas. A espécie evidenciou aptidão para solos de textura arenosa, bem drenados, pH ácido, de baixa fertilidade e acentuado teor e saturação de alumínio (m\%).

Não obstante fatos e dados observados, estudos adicionais de fertilidade e de outros componentes do ecossistema ambiental são de grande relevância e aprimoramento, como instrumentos preditivo do crescimento e desenvolvimento da espécie, com uso relevante em estratégias futuras de conservação ou em programas de melhoramento.

\section{Referências}

Ab'saber, A. (2003). Os domínios da natureza no Brasil: potencialidades paisagísticas. Atelier Editorial. 
Cruz, C. D., \& Souza Carneiro, P. C. (2006). Modelos biométricos aplicados ao melhoramento genético (No. 575.1015195). Universidade Federal de Viçosa.

Empresa Brasileira de Pesquisa Agropecuária (2018). Centro Nacional de Pesquisa de Solo. Sistema brasileiro de classificação de solos (5a ed.). Embrapa Produção de Informações, Brasília, DF, Brasil.

Franco, C. R. P., Alves, P. B., Andrade, D. M., Jesus, H. C. R., Silva, E. J. S., Santos, E. A. B., Antoniolli, A. R., \& Quintans Júnior, L. J. (2011a). Essential oil composition and variability in Hyptis fruticosa. Brazilian Journal of Pharmacognosy, 21: 24-32.

Fundação Instituto Brasileiro de Geografia e Estatística (2019). Informações ambientais.

Geliwania, A. B et al. (2020). Potencial toxicológico e caracterização físico-química e fitoquímica da Garcínia camboja. Research, Society and Development v. 9, n. 4.

Gobbo-Neto, L., \& Lopes, N. P. (2007). Plantas medicinais: fatores de influência no conteúdo de metabólitos secundários. Química. Nova, 30(2), 374-381,

Harley, R. M. (2014) Eplingiella brightoniae, a new species of Hyptidinae (Lamiaceae: Ocimeae) from Northern Bahia, Brazil, Kew Bulletin, 69(4).

Harley, R. M. (2012).Checklist and key of genera and species of the Lamiaceae of the Brazilian Amazon. Rodriguésia, 63(1), 129-144.

Instituto Nacional de Meteorologia (2019) - Históricos de dados meteorológicos.

Lemes, G. F., Ferri, P. H., \& Lopes, M. N.(2011) Constituintes químicos de Hyptidendron canum (Pohl ex Benth.) R. Harley (Lamiaceae). Química Nova. Sociedade Brasileira de Química, 34(1), 39-42.

Magnago, L. F. S., Martins, S. V., Schaefer, C. E. G., \& Neri, A. V. (2010). Gradiente fitofisionômico-edáfico em formações florestais de Restinga no Sudeste do Brasil. Acta Botanica Brasilica, 24(3), 734-746.

Meira, M. R. et al.(2017). Ecogeography of Lippia rotundifolia Cham. In Minas Gerais, Brazil. Cienc. Rural, Santa Maria, 47(8), e20160921.

Melo, M. P.(2012). Conservação de Lippia sidoides Cham do norte de Minas Gerais e Vale do Jequitinhonha: localização, coleta, ecogeografia, crescimento, modo de reprodução e divergência genética.100f.(Dissertação Mestrado em Agroecologia) - Universidade Federal de Minas Gerais, Montes Claros, Brasil.

Mendes, A. D. R. et al.(2015). Ecogeography populations of Cordia (Varronia curassavica Jacq.) in North and Jequitinhonha Valley in Minas Gerais, Brazil. Ciência Rural, 45(3), 418-424.

Oliveira, L. O., Vieira, I. J. C., Maia, J. T. L. S., \& Martins, E. R. (2009). Estudo ecogeográficos da poaia [Psychotria ipecacuanha (Brot.) Stokes].

Ross, J. L. S. (2014). (Org.), Geografia do Brasil, EDUSP - Editora da Universidade de São Paulo, São Paulo. Brasil.540 p.

Ribeiro, A. C., Gontijo, P. T. G., \& Alvarez V. H. V. (1999). Recomendações para o uso de corretivos e fertilizantes em Minas Gerais - $5^{a}$ aproximação. Viçosa, Comissão de Fertilidade do Solo do Estado de Minas Gerais, 359p.

Dos santos, H. G. et al.(2018). Sistema brasileiro de classificação de solos. Embrapa, 2018.

Silva, A. C. (2015). Caracterização agronômica, molecular e fitoquímica de Eplingiella Harley \& JFB PASTORE.125 f. Tese (Doutorado em Recursos Genéticos Vegetais) - Universidade Estadual de Feira de Santana - UEFS, Feira de Santana, Ba, Brasil.

Silva, W. J., Dória, G. A. A., Maia, R. T., Nunes, R. S., Carvalho, G. A., Blank, A. F., \& Cavalcanti, S. C. H. (2008). Effects of essential oils on Aedes aegypti larvae: alternatives to environmentally safe insecticides. Bioresource technology, 99(8), 3251-3255.

Silva, A. B. L et al.(2006). Avaliação do efeito antinociceptivo e da toxicidade aguda do extrato aquoso da Hyptis fruticosa Salmz.ex Benth. Revista Brasileira de Farmacognosia. 16(4), 475-479.

Souza, G. A., Queiroz, J. M. R., Anjos, O. F., Santos, E. A. M., Martins, E. R., Fernandes, L. A., \& Costa, C. A. (2008). Levantamento ecogeográficos de Dimorphandra mollis Benth. (Leguminosae-Caesalpinioideae) no Norte de Minas Gerais. Revista Brasileira de Plantas Medicinais, Botucatu, 10(4), 51-62.

Teixeira, P. C., Donagemma, G. K., Fontana, A., \& Teixeira, W. G., (2017). Manual de Métodos de Análise do Solo, EMBRAPA. 